\title{
Anomalous dielectric relaxation of water molecules at the surface of an aqueous micelle
}

\author{
Subrata Pal \\ Solid State and Structural Chemistry Unit, Indian Institute of Science, Bangalore 560012, India \\ Sundaram Balasubramanian ${ }^{\text {a) }}$ \\ Chemistry and Physics of Materials Unit, Jawaharlal Nehru Centre for Advanced Scientific Research, \\ Jakkur, Bangalore 560064, India \\ Biman Bagchib) \\ Solid State and Structural Chemistry Unit, Indian Institute of Science, Bangalore 560012, India
}

\begin{abstract}
Dielectric relaxation of aqueous solutions of micelles, proteins, and many complex systems shows an anomalous dispersion at frequencies intermediate between those corresponding to the rotational motion of bulk water and that of the organized assembly or macromolecule. The precise origin of this anomalous dispersion is not well-understood. In this work we employ large scale atomistic molecular dynamics simulations to investigate the dielectric relaxation (DR) of water molecules in an aqueous micellar solution of cesium pentadecafluorooctanoate. The simulations clearly show the presence of a slow component in the moment-moment time correlation function $\left[\Phi_{M}^{W}(t)\right]$ of water molecules, with a time constant of about $40 \mathrm{ps,} \mathrm{in} \mathrm{contrast} \mathrm{to} \mathrm{only} 9 \mathrm{ps}$ for bulk water. Interestingly, the orientational time correlation function $\left[C_{\mu}(t)\right]$ of individual water molecules at the surface exhibits a component with a time constant of about $19 \mathrm{ps}$. We show that these two time constants can be related by the well-known micro-macrorelations of statistical mechanics. In addition, the reorientation of surface water molecules exhibits a very slow component that decays with a time constant of about $500 \mathrm{ps}$. An analysis of hydrogen bond lifetime and of the rotational relaxation in the coordinate frame fixed on the micellar body seems to suggest that the 500 ps component owes its origin to the existence of an extended hydrogen bond network of water molecules at the surface. However, this ultraslow component is not found in the total moment-moment time correlation function of water molecules in the solution. The slow DR of hydration water is found to be well correlated with the slow solvation dynamics of cesium ions at the water-micelle interface.
\end{abstract}

\section{INTRODUCTION}

Understanding the dynamics of water molecules at the surfaces of self-organized assemblies and complex biological macromolecules in aqueous solution has been a subject of considerable interest for a long time, being pursued by physicists, chemists, and biologists alike. The reason for such a sustained interest is twofold. First, water at the interface is expected to play an important role in the dynamics and function of the self-assembly or the macromolecule. Thus, a study of the rotational and translational dynamics of surface water should provide valuable information on several fronts. Second, since the dynamics of water molecules at heterogeneous surfaces is not easily and directly amenable to experimental studies, progress has been rather slow. Among various experimental techniques, the study of dielectric relaxation of aqueous proteins, deoxyribose nucleic acide and micellar solutions has proved to be rather fruitful. In fact, the crucial understanding that the dynamics of water at the surfaces of biological macromolecules and of self-

\footnotetext{
a)Electronic mail: bala@jncasr.ac.in

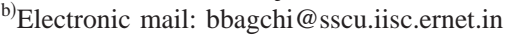

organized assemblies are indeed different from the bulk was first elucidated by dielectric relaxation (DR) experiments and computer simulations of aqueous protein solutions and colloids. ${ }^{1-7}$ In a macromolecular solution, one usually finds three significant time constants in the relaxation spectrum, (i) a 10 ps component belonging to bulk water, (ii) a $10-100 \mathrm{~ns}$ component that can be attributed to the reorientation of the macromolecule, and (iii) a "universal" component in the range of 40-60 ps whose origin is the subject of the present study. This weak but distinct dispersion, sometimes called the $\delta$ dispersion, has often been attributed to the water molecules at the surface of the macromolecule. ${ }^{1,6}$

Several recent studies have addressed the issue of polar solvation dynamics in aqueous micelles, lipid vesicles, and protein solutions, using fluorescent probes located near the surface of the macromolecule or organized assembly. ${ }^{1-12}$ In all these solvation dynamics experiments, a slow component was observed. In some cases, this slow component was found to decay with a time constant in the range of 20-40 ps. In some cases, even a slower solvation, with time constants ranging from several hundred picoseconds to a few nanoseconds have been reported. ${ }^{10} \mathrm{~A}$ detailed quantitative 
understanding of the origin of such slow decays remains to be achieved.

A phenomenological theory developed recently, proposed to explain the slow dynamics. ${ }^{1,13}$ The observed time scale of the surface water was proposed to arise from a dynamical equilibrium between "bound" and "free" water at the surface of macromolecules. This theory predicts the slowing down of dielectric relaxation when the binding energy of interfacial water with the hydrophilic, polar head group of say, a micelle (or the amino acids exposed to water in a protein solution) is strong (larger than $2-3 k_{B} T$ ). Specifically, the long time decay of the dielectric relaxation is equal to the inverse of the rate of the transition between free and bound water.

Computer simulations have played an important role in providing insights into microscopic processes and phenomena that complement experimental data. Several groups have reported detailed simulations of orientational relaxation and translational diffusion of water molecules; on micellar surfaces, ${ }^{14-17}$ inside reverse micelles ${ }^{18,19}$ and also at the surface of aqueous proteins. ${ }^{20}$ Our detailed simulations and the ones carried out by Berkowitz and co-workers have clarified many aspects of water dynamics at the surfaces of selfassemblies. In particular, these simulations have clearly shown that the rotational dynamics and translational motion of the surface water molecules become significantly perturbed by the presence of the surface. Because of the relative small size of the systems studied in these simulations, rather long molecular dynamics (MD) runs were possible to carry out which was essential to detect the slow decay components.

In the present paper we use the same atomistic MD simulation to study the contribution of only the water molecules to the DR in this system. The objective is to understand the collective dynamics of the water molecules that contribute to dielectric relaxation, and its relation to the single-particle, reorientational motion of interfacial water molecules. The interesting new result is the finding that water molecules indeed show a DR in the 40 ps time scale. Equally important is the fact that the simulations unequivocally show that it is the slow orientational relaxation of the water molecules at the surface which is responsible for the 40 ps component in the DR. This DR component fits elegantly the macro-microrelation ${ }^{21,22}$ that has been developed to understand the relationship between the single particle orientational relaxation and DR. In addition, we find the existence of an ultraslow component with a time constant of about $500 \mathrm{ps}$ in the orientational relaxation of the surface water molecules. Analysis of the origin of this slow component leads to the conclusion that this slow rotational relaxation is due to the existence of extensive hydrogen bond network at the surface with the polar head groups of the micelle.

The rest of the paper is organized as follows. In the next section we discuss the simulation details. In Sec. III, we present the results and discuss their importance. Section IV contains the concluding remarks.

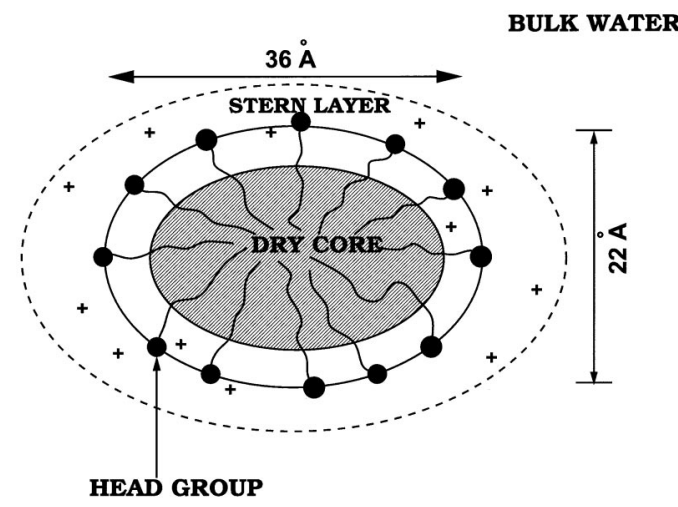

FIG. 1. Schematic illustration of the average configuration of the simulated CsPFO micelle. The micelle can be represented as general ellipsoidal in shape. Its extent in the third direction, perpendicular to the plane of the paper, is estimated to be around $46 \AA$. The region outside the "dry core" contains water. Water molecules present within a distance of $4.35 \AA$ from the carbon atom of the head group of any surfactant molecule, constitute the interfacial region in our discussion. The plus signs denote counterions.

\section{DETAILS OF SIMULATION}

The surfactant in our simulations is cesium pentadecafluorooctanoate (CsPFO). The $\mathrm{CsPFO}-\mathrm{H}_{2} \mathrm{O}$ system has been well studied experimentally, ${ }^{23,24}$ and the critical micelle concentration is around 0.02 weight fraction of $\mathrm{CsPFO}$ in water. Our molecular dynamics simulations were carried out in the NVT ensemble for an aggregate of $62 \mathrm{CsPFO}$ molecules in 10562 water molecules at $300 \mathrm{~K}$. The potential for water molecules is the extended simple point charge (SPC/E) model, ${ }^{25}$ the counterions carry a unit positive charge, which is compensated by a $+0.4 e$ charge on the carbon of the octanoate head group and a $-0.7 e$ charge on each of the oxygens of the head group. ${ }^{26}$ The surfactant is modeled with explicit fluorine atoms and interactions between the fluorocarbon tails were obtained from the work of Sprik et al. on polytetrafluoroethylene. ${ }^{27}$ Other details of the potential parameters are provided elsewhere. ${ }^{14}$ The equations of motion were integrated with the reversible reference system propagator algorithm scheme ${ }^{28}$ using the PINY-MD package ${ }^{29}$ with an outer time step of 4 fs. Coulombic interactions were treated using the particle mesh Ewald method. The linear dimensions of the simulation cell at $300 \mathrm{~K}$ were 80.6, 80.6, and $52.1 \AA$. Details of the simulations can also be found elsewhere. ${ }^{14-16,30,31}$ The analyses reported here were carried out from different sections of a 3 ns trajectory. The results pertaining to interfacial water are compared to a simulation of bulk water that contained 256 molecules of the SPC/E type at a temperature of $300 \mathrm{~K}$ and a density of $1.02 \mathrm{~g} / \mathrm{cm}^{3}$.

In order to aid the discussion that follows, we provide a schematic illustration of the micelle in Fig. 1.

\section{RESULTS AND DISCUSSION}

\section{A. Dielectric relaxation}

DR measures the complex dielectric function, $\epsilon(\omega)$, that can be decomposed into real and imaginary parts as ${ }^{32-34}$

$\epsilon(\omega)=\epsilon^{\prime}(\omega)-i \epsilon^{\prime \prime}(\omega)$, 
where $\epsilon^{\prime}(\omega)$ and $\epsilon^{\prime \prime}(\omega)$ are the real part (permittivity factor) and imaginary (dielectric loss) parts, respectively. The total dipole moment of the system, at any given time $t, \mathbf{M}(t)$, is defined as

$$
\mathbf{M}(t)=\sum_{i=1}^{N} \boldsymbol{\mu}_{i}(t),
$$

where $N$ is the total number of dipolar molecules and $\boldsymbol{\mu}_{i}$ is the dipole moment vector of the $i$ th molecule. The complex dielectric function $\epsilon(\omega)$ is given by the following exact relation: ${ }^{33,32}$

$$
\begin{aligned}
\frac{\epsilon(\omega)-\epsilon(\infty)}{\epsilon(0)-\epsilon(\infty)} & =\int_{0}^{\infty} d t e^{-i \omega t}\left[-\frac{d \Phi_{M}(t)}{d t}\right] \\
& =1-i \omega \int_{0}^{\infty} d t e^{-i \omega t} \Phi_{M}(t),
\end{aligned}
$$

where $\epsilon(0)$ and $\epsilon(\infty)$ are the limiting low- and high-frequency permittivities, respectively. $\Phi_{M}(t)$ is the normalized time auto correlation function of the system's total dipole, $\mathbf{M}$, and is defined as

$$
\Phi_{M}(t)=\frac{\langle\mathbf{M}(t) \cdot \mathbf{M}(0)\rangle}{\langle\mathbf{M}(0) \cdot \mathbf{M}(0)\rangle} .
$$

The total moment of the system can be split into contributions from the water, the micelle, and the ions. Thus, $\Phi_{M}(t)$ will contain terms that are self-correlation functions, such as, water-water, and crosscorrelations, such as water-micelle. The calculation of the relaxation of the total moment of the micellar solution from a finite sized simulation containing only one micelle, is wrought with interpretational difficulties, chief of which is the absence of intermicellar contributions. In addition, the treatment of ions in DR need to be handled with care too. However, we focus here on the contribution from water alone to the DR of the system, i.e., we calculate

$$
\Phi_{M}^{W}(t)=\frac{\left\langle\mathbf{M}_{\mathbf{W}}(t) \cdot \mathbf{M}_{\mathbf{W}}(0)\right\rangle}{\left\langle\mathbf{M}_{\mathbf{W}}(0) \cdot \mathbf{M}_{\mathbf{W}}(0)\right\rangle},
$$

where $M_{W}(t)$ is similar to Eq. (2), but with the sum extending only over atoms of all water molecules in the system. Figure 2 shows the total moment-moment time correlation function (TCF), $\Phi_{M}^{W}(t)$, of water (only), both in the presence (solid line) and in the absence of the micelle in the simulation box, i.e., in bulk or neat water. The TCF $\Phi_{M}^{W}(t)$ was fitted to a form containing a sum of three exponential terms

$$
\Phi_{M}^{W}(t)=C_{1} e^{-t / \tau_{1}}+C_{2} e^{-t / \tau_{2}}+C_{3} e^{-t / \tau_{3}}
$$

to get the time constants and amplitudes which are presented in Table I. Note that the $\Phi_{M}^{W}(t)$ of neat water decays exponentially with a time constant of $9 \mathrm{ps}$ for SPC/E water, in good agreement with the experimental estimate of 8.3 ps. The value of the computed static dielectric constant of neat water in the absence of micelle is 67 (Ref. 35) while the value of the same with contributions only from the water molecules in our micellar system is found to be 68 .

The presence of the $41 \mathrm{ps}$ component in the dielectric relaxation of the micellar water can be traced back to the

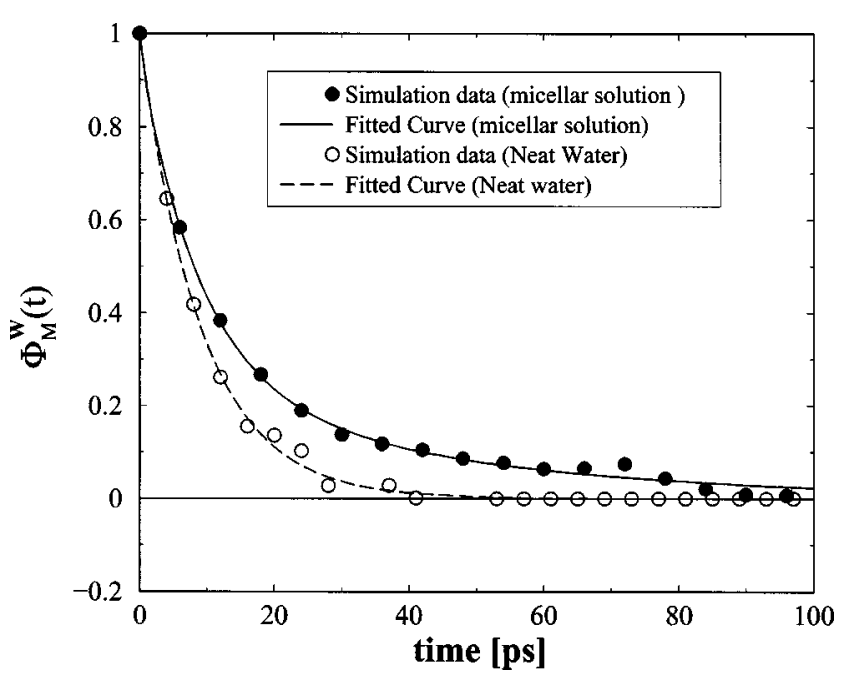

FIG. 2. Time dependence of the normalized total moment-moment time correlation function $\left[\Phi_{M}^{W}(t)\right]$ for the water molecules, both in the micellar solution (solid line) and in neat water. Circles are the simulation data and the continuous line is a multiexponential fit. Data points in the former are shown infrequently for clarity.

dynamic exchange of water molecules between its bound and the free states at the interface. We shall return to this point again later.

\section{B. Relationship with orientational time correlation function of interfacial water}

The interfacial region of water around the micelle can be defined using the pair correlation function of water molecules with respect to the carbon atoms of the head group. The first minimum in this function is found to be at 4.35 $\AA .{ }^{14,30}$ In Fig. 3, we show the single particle dipolar orientational relaxation $\left[C_{\mu}(t)\right]$ of water molecules near the surface (within $4.35 \AA$ from any surfactant head group), comparing it to its relaxation in bulk. Note the pronounced slower decay of the former. In Table I, we present time con-

TABLE I. Parameters of multiexponential fits to the normalized total moment-moment time correlation function $\left[\Phi_{M}^{W}(t)\right]$ of all the water molecules in the micellar system, that for the normalized dipolar time correlation function $\left[C_{\mu}(t)\right]$ of the interfacial water molecules (staying within 4.35 $\AA$ from the any polar head group of the micelle), and for the solvation time correlation function $\left[C_{S}(t)\right]$ of $\mathrm{Cs}^{+}$ions in the micellar solution (see Ref. $15)$.

\begin{tabular}{cccc}
\hline \hline \multirow{4}{*}{$\Phi_{M}^{W}(t)$} & & Time constant $(\mathrm{ps})$ & Amplitude $(\%)$ \\
& $\tau_{1}$ & 0.4 & 3.6 \\
& $\tau_{2}$ & 8.9 & 70.0 \\
$C_{\mu}(t)$ & $\tau_{3}$ & 41.0 & 26.4 \\
& $\tau_{1}$ & 0.4 & 14.0 \\
& $\tau_{2}$ & 5.0 & 29.0 \\
& $\tau_{3}$ & 19.5 & 28.0 \\
$C_{S}(t)$ & $\tau_{4}$ & $507.0^{\mathrm{a}}$ & 29.0 \\
& $\tau_{1}$ & 0.3 & 15.0 \\
& $\tau_{2}$ & 3.9 & 15.0 \\
& $\tau_{3}$ & 56.0 & 70.0 \\
\hline \hline
\end{tabular}

${ }^{a}$ The value of $507 \mathrm{ps}$ as the time constant for the ultraslow component in the $C_{\mu}(t)$ is only nominal, as the time correlation function can be calculated reliably only up to $150 \mathrm{ps}$. 


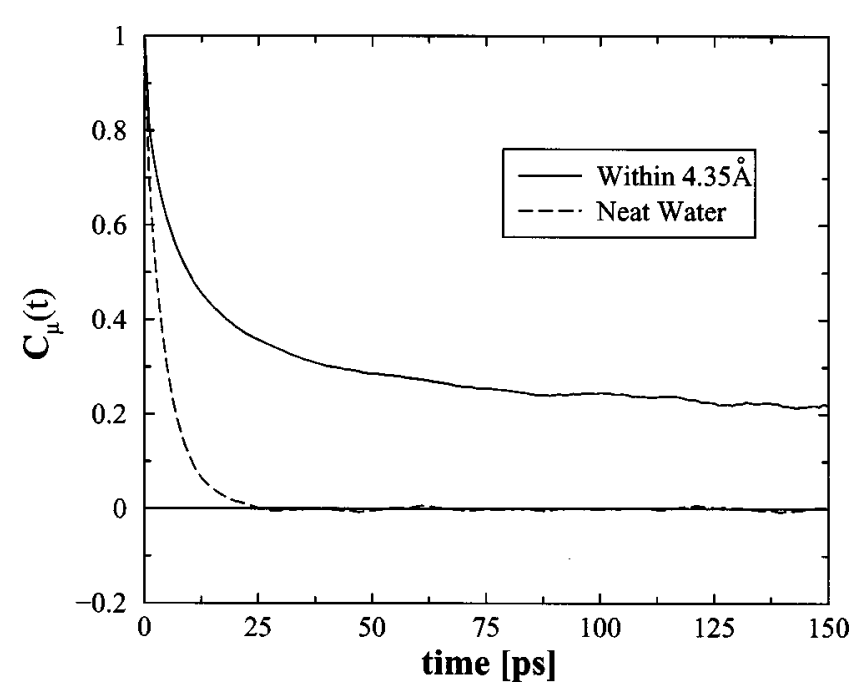

FIG. 3. Dipole-dipole time correlation function $\left[C_{\mu}(t)\right]$ for the interfacial water molecules within $4.35 \AA$ away from any polar head group of the micelle, and for bulk water, calculated in the laboratory frame.

stants obtained by fitting the average orientational correlation function of water molecules in the hydration layer to a multiexponential form. Here, one finds an intermediate slow decay with a time constant of about 19 ps which is again much slower than that for neat water which is about 4 ps and the decay in neat water is single exponential. The orientational time correlation function for hydration water exhibits an additional slow (or, rather ultraslow) decay of time constant of 507 ps with an amplitude of $30 \%$. We have discussed elsewhere that this ultraslow component is due to the strongly bound water molecules that are found to form two hydrogen bond with the polar head group of the micelle. ${ }^{36,37}$ However, a careful analysis shows that the earlier conclusion was only partly correct, as discussed in more detail later.

It is important to correlate the slow decay of the orientational time correlation function with the slow decay observed in the total moment-moment time correlation function. Such a relation is known in the statistical mechanical literature as a macro-microrelation because the total moment is a macroscopic quantity while the single particle correlation is a microscopic quantity. Several such relations have been proposed in studies of dielectric relaxation. ${ }^{38}$ In all these relations, the moment-moment time correlation function time constant $\tau_{M}$ is predicted to be considerably larger than the single particle orientational correlation time, $\tau_{R}$. A simple continuum model based form of this relation was proposed by Powles and is given by ${ }^{21,38}$

$$
\tau_{R}=\frac{2 \epsilon_{0}+\epsilon_{\infty}}{3 \epsilon_{0}} \frac{\tau_{M}}{g_{K}},
$$

where $g_{K}$ is the well known Kirkwood $g$ factor. $g_{K}$ for water is equal to 2.8 and $\epsilon_{0}$ ( simulated $) \simeq 70$. We take $\epsilon_{\infty} \simeq 5$, since the above Powles relation was based on the assumption of a single exponential Debye behavior. When these estimates are used in the above expression, we get a ratio, $\tau_{M} / \tau_{R} \simeq 4$. A microscopic relation (involving pair correlation function) also gives values in the similar range. ${ }^{22,38}$ Thus, it is fair to conclude that the 19 ps component in the orientational relaxation in indeed responsible for the $41 \mathrm{ps}$ component observed in the dielectric relaxation.

What is the origin of the ultraslow, $500 \mathrm{ps,} \mathrm{component}$ (with $30 \%$ amplitude) in the orientational relaxation of the water molecules in the hydration layer? One can attempt to estimate this long time component from the inverse of the rate of transition from bound to free states of water $\left(k_{\mathrm{bf}}\right)$, $\tau_{R}^{\text {long }} \simeq k_{\mathrm{bf}}^{-1} \cdot{ }^{13}$ However, this gives a much smaller value. In fact, a numerical study of the bound to free transition rates also confirm that the 500 ps component cannot be explained in terms of transition between bound and free states, at least for the present micellar system.

In order to unravel the origin of this ultraslow component, we have calculated the TCF of the water dipole in the time dependent, principal axes frame of the micelle, rather than in the laboratory frame. This procedure should remove contributions, if any, from those water molecules which are bound to the micellar surface (sticky molecules) and which rotate along with the overall rotation of the micelle and thus contribute to the ultraslow reorientation of water dipole observed from the laboratory frame. Here, the principal axes of the micelle were obtained as the eigenvectors of the instantaneous moment of inertia matrix of the micelle. Three distinct eigenvalues were obtained, whose time evolution are shown in Fig. 4(a). The simulated micelle can thus be described as a general ellipsoid in shape. The central core of the micelle contains surfactants arranged in the form of a bilayer and the eigenvector corresponding to the largest eigenvalue was found to be oriented along this direction, in accord with the moment of inertia expected for ellipsoids. The extent of the micelle in this direction is approximately twice the end-to-end length of the surfactant. To obtain an idea of the overall rotation of the micelle, we plot in Fig. 4(b), the time autocorrelation functions of the principal axes themselves. We find that $\mathbf{P}_{1}$ and $\mathbf{P}_{3}$ axes do not reorient in any significant manner over the time span of 1-2 ns, while the $\mathbf{P}_{2}$ axis reorients in a time scale of about $500-800 \mathrm{ps,} \mathrm{a}$ value that is still large on the time scales of the reorientation of interfacial water molecules.

In Fig. 5(a), we show the distribution of the angle made by the end-to-end vector of the surfactant, defined as the vector connecting the first and the last carbon atom, with the principal axes of the micelle. It is evident that a large fraction of the surfactants are oriented along the $\mathbf{P}_{3}$ axis. It would thus be natural to expect that the bisector of the polar head group of the surfactants and their vector sum too to be oriented along this direction. We present the orientational distribution of the total dipole moment of the micelle (that arises only from the charged head groups of the surfactant), calculated with respect to its centroid, in Fig. 5(b). There is a clear preference for the total dipole moment of the micelle to be oriented along the $\mathbf{P}_{3}$ axis. We estimate its magnitude to be around $9 \mathrm{D}$.

This anisotropy in the internal structure of the micelle and consequently, in the orientation of the polar head groups (PHG) and in the total dipole moment of the micelle, influences the orientation of the dipole vector of the water molecules. In Fig. 6, we show the distribution of the angles made 


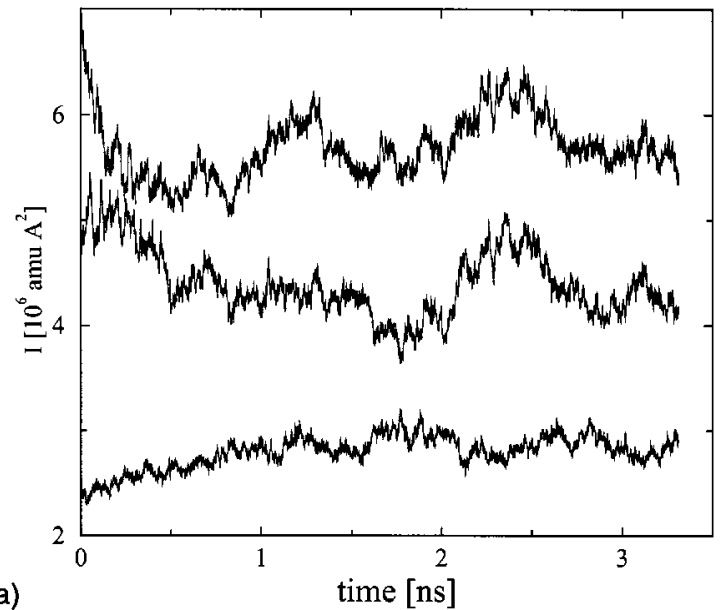

(a)

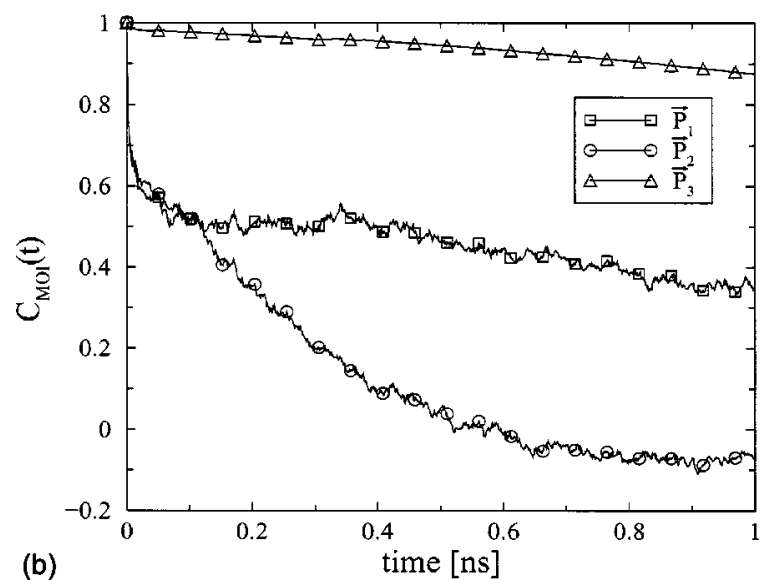

FIG. 4. (a) Eigenvalues of the moment of inertia matrix calculated from the atoms constituting the micelle as a function of time. The largest eigen value corresponds to the smallest extent of the micelle, i.e., along its bilayer core, and corresponds to the $\mathbf{P}_{3}$ axis described in Fig. 4(b). (b) Time auto correlation functions, $C_{\mathrm{MOI}}(t)$, of the eigen vectors (principal axes) of the micelle, represented in the laboratory frame.

by the dipole vectors of water molecules present in two interfacial regions, with the principal axes of the micelle. The water molecules that are present within $4.35 \AA$ away from the head groups of the surfactants, show a noticeable preference to have their dipole moments oriented along the $\mathbf{P}_{3}$ axis of the micelle, while water molecules that are present between 4.35 and $9 \AA$ away from the micellar surface exhibit an isotropic distribution of dipole vectors. We believe that this marginal, preferential orientation of water dipoles at the interface is caused by their interaction with the effective dipole moment of the micelle that is oriented along the $\mathbf{P}_{3}$ axis. This dipole-dipole interaction decays with distance away from the center of mass of the micelle, and thus is ineffective in inducing a similar anisotropy in the orientation of water molecules present in the second interfacial region.

It is thus natural to expect that the anisotropy in orientation will lead to different rates of reorientation of the water dipole along different axes. We show, in Fig. 7(a), the reorientational TCFs of the components of the water dipole vector along the three principal axes of the micelle for water molecules in the second interfacial shell, i.e., between 4.35 and 9 $\AA$ away from the micellar surface. All three components de-
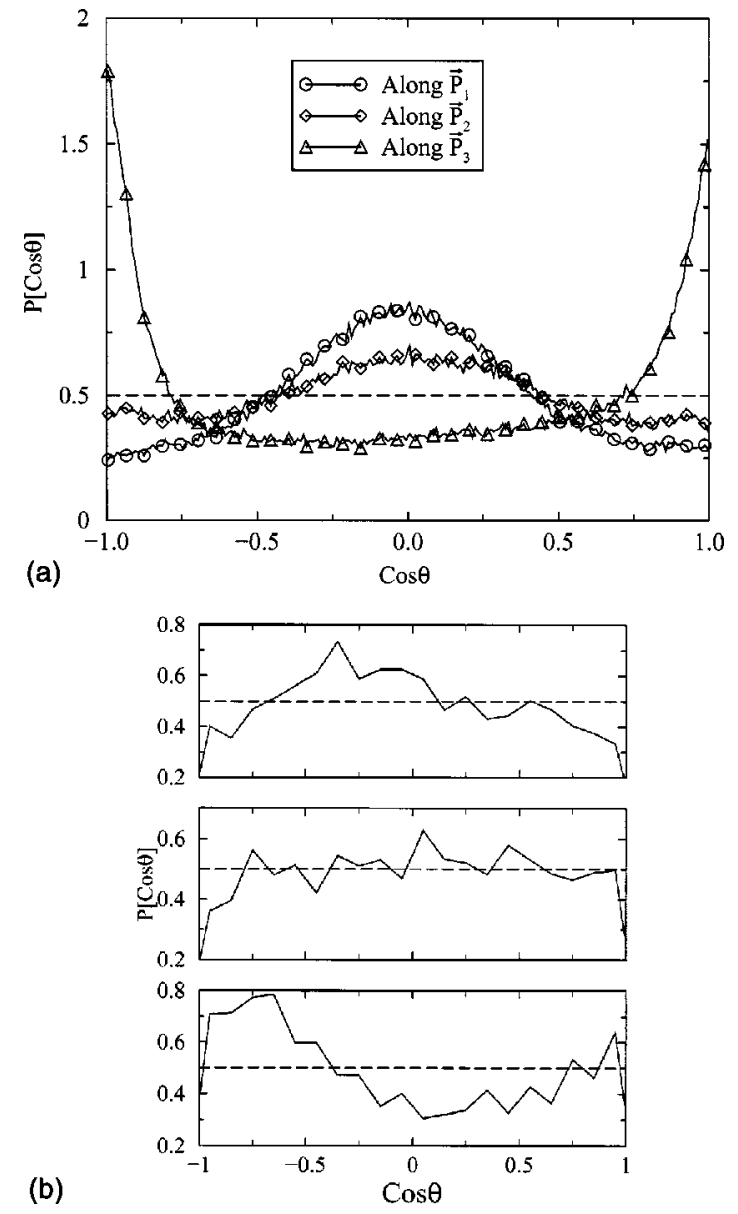

(b)

FIG. 5. (a) Distribution of the cosine of the angle, $\theta$, made by the end-to-end vector of the surfactants with the principal axes of the micelle. A uniform value of 0.5 (dashed line) is expected for an isotropic distribution. (b) Distribution of the cosine of the angle, $\theta$, made by the total dipole moment vector of the micelle, calculated with respect to its centroid, with the principal axes of the micelle. Top to bottom: Along $\mathbf{P}_{1}, \mathbf{P}_{2}$, and $\mathbf{P}_{3}$ axes. The dashed line with a value of 0.5 is the expected value for an isotropic distribution.

cay significantly within $50 \mathrm{ps,}$, and the relaxation behavior is isotropic, as is to be expected from the orientational distribution of the dipole vectors shown in Fig. 6. However, the data for water molecules closest to the micellar surface, i.e., within $4.35 \AA$ of the head groups is vastly different. This is shown in Fig. 7(b). We find that the dipole components along $\mathbf{P}_{1}$ and $\mathbf{P}_{2}$ decay to a zero value in about $150 \mathrm{ps}$, while that along the $\mathbf{P}_{3}$ axis does not decay. These time correlation functions can decay due to two reasons. One, from the inherent reorientation of the water dipole along that axis, and two, from the reorientation of the principal axes themselves in space. The contribution of the latter is expected to be minimal, based on the data presented in Fig. 4(b), and also from the fact that the components of water dipole do not reorient even with respect to a time independent laboratory frame (not shown).

Note that one should be careful about the possibility that at least a part of the slowness in the decay of the TCF of $\mathbf{P}_{3}$ [Fig. 4(b)] might be an artifact; the length of the simulation cell is $82 \AA$ each in the Cartesian (laboratory frame) $x$ and $y$ axes, while it is only $52 \AA$ in the $z$ axis. The initial configu- 


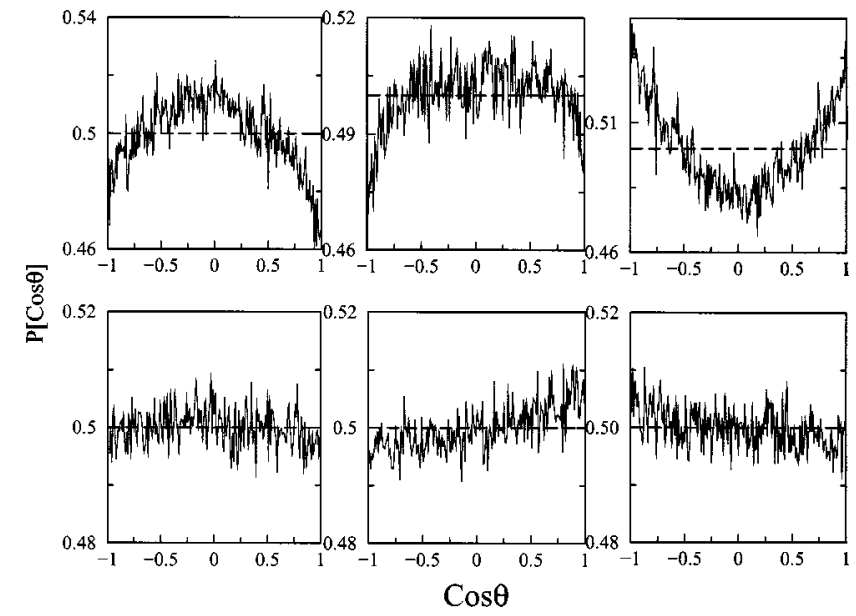

FIG. 6. Distribution of cosine of the angle $\theta$, made by the dipole vectors of water molecules present in two interfacial regions, with the principal axes of the micelle. Top panel: water molecules within $4.35 \AA$ away from any surfactant head group. Bottom panel: water molecules between 4.35 and $9 \AA$ away from any surfactant head group. The plots describe, from left to right, the angle distribution with respect to $\mathbf{P}_{1}, \mathbf{P}_{2}$, and $\mathbf{P}_{3}$ axes. The dashed line with a value of 0.5 is the expected value for an isotropic distribution.

ration of the micelle was setup such that the bilayer region, with an extent of about $24 \AA$ was oriented along the $z$ axis. Based on visualization of equilibrated configurations, we estimate the size of the micelle along the $\mathbf{P}_{1}$ and $\mathbf{P}_{2}$ axes to be approximately 46 and $36 \AA$, respectively. The micelle may thus be constrained to maintain its $\mathbf{P}_{3}$ axis aligned, to some extent with the $z$ axis, due to the shorter box length along that direction. Thus, a part of the long time (hundreds of picoseconds) decay of the TCF of the interfacial water dipole along the $\mathbf{P}_{3}$ axis might be an artifact arising from the inability of the $\mathbf{P}_{3}$ axis to reorient.

It is certainly natural to expect that the time scales of reorientation of the $\mathbf{P}_{3}$ axis be much larger than that needed to reorient the $\mathbf{P}_{1}$ and $\mathbf{P}_{2}$ axes, based on their lengths. Therefore, if the slowness of the orientation of the water molecules owes its origin to the slow rotation of the $\mathbf{P}_{3}$ axis, then this slowness will have to persist even when the rotation of this axis is allowed. This adds support to the claim that the ultraslow component in the reorientation of dipoles of interfacial water is genuine. In addition, the time scales of relaxation of the water dipole is observed to be much smaller (few hundreds of picoseconds) than the time constant of reorientation of the micellar axes (around $1 \mathrm{~ns}$ or more). This too leads us to believe that the ultraslow component is unlikely to be purely an artifact. The finite duration of the simulated TCFs, arising out of the limits on the residence times of water molecules in the interfacial layer, prevent us from determining exactly the time constant of this ultraslow relaxation component. The reduced simulation cell length along the $z$ direction also casts an uncertainty in the amplitude of this component. However, its existence and the rationale for it, explained earlier in terms of the anisotropy in the orientation of interfacial water dipoles that arises from the intrinsic structure of the micelle, cannot be doubted. The decay of the water dipole components along the $\mathbf{P}_{1}$ and $\mathbf{P}_{2}$ axes can be fitted to multiexponential functions with time constants of
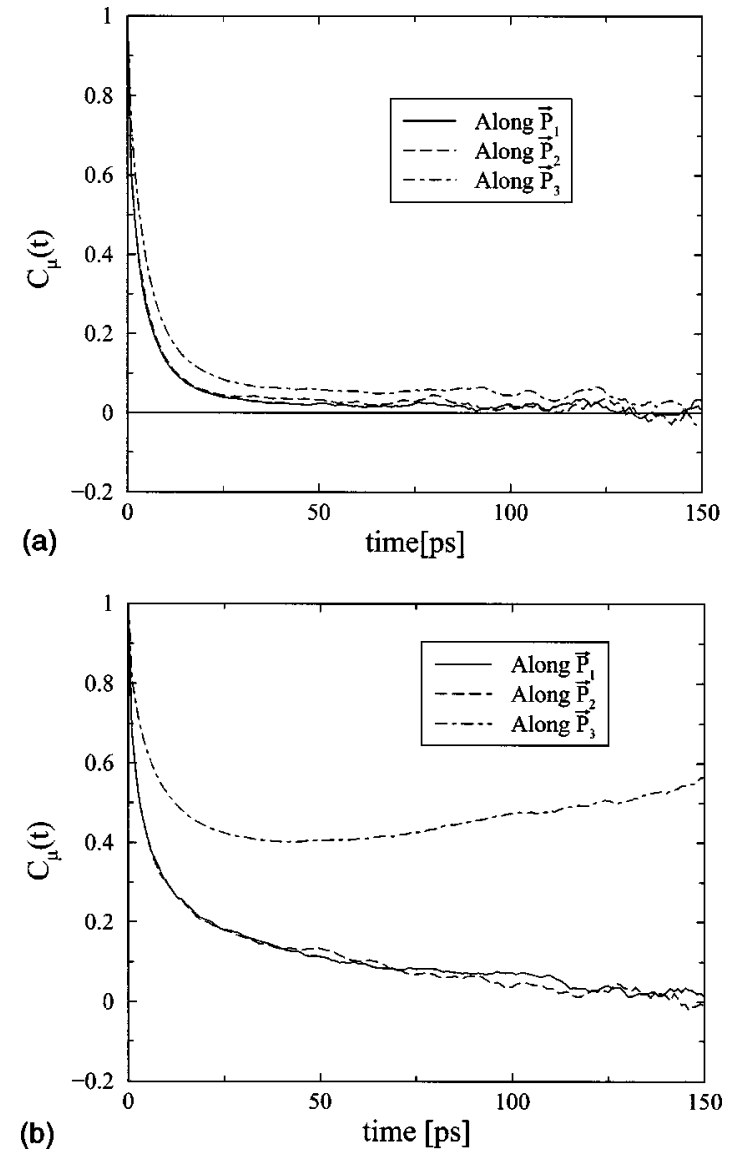

FIG. 7. (a) Time correlation function of the components of the dipole moment vector of water molecules that lie between 4.35 and $9 \AA$ away from the polar head groups of the micellar surface. $\mathbf{P}_{1}, \mathbf{P}_{2}$, and $\mathbf{P}_{3}$ represent the principal axes of the micelle. (b) Time correlation function of the components of the dipole moment vector of water molecules within the first coordination shell of the polar head groups of the micelle. $\mathbf{P}_{1}, \mathbf{P}_{2}$, and $\mathbf{P}_{3}$ represent the principal axes of the micelle.

0.84 ps (37.2\%), 7.2 ps (38.4\%), and 68.2 ps (24.2\%), while that along the $\mathbf{P}_{3}$ axis yields two time constants with values 0.74 , and $8.4 \mathrm{ps}$, and a third component whose value cannot be determined as this ultraslow decay could not be captured adequately within the limited duration of the TCF.

Let us summarize the earlier analyses. We have uncovered that there is a noticeable anisotropy in the orientation of the surfactants in the micelle. We believe that the slowness of the rotation of the $\mathbf{P}_{3}$ axis could be partly responsible for the slowness in surface water rotation. Note that this is a direct consequence of the shape of the micelle. A part of the slow reorientation of the interfacial water also arises from its interaction with the effective dipole moment of the micelle that is oriented preferentially along the $\mathbf{P}_{3}$ axis. We shall come back to this point again later where we connect this to an analysis involving mode coupling theory.

Another interesting aspect of the puzzle is the absence of any contribution of this 507 ps component to the relaxation of the total moment time correlation function of hydration water (see Table I). Thus, this contribution gets fully cancelled out in the total moment which is possible only if the slow component comes from bound water molecules which are expected to be arranged in the opposite directions on the 
diametrically opposite surfaces of the micelle because the polar hydrogen groups of the surfactant are always projected outwards, that is, towards the opposite sides.

Our previous analysis has shown that in CsPFO micelle, only about $10 \%$ of the interfacial water molecules are doubly hydrogen bonded to the micellar surface and, the majority, $80 \%$, are singly hydrogen bonded to the negatively charged oxygen atoms of the polar head groups. ${ }^{36}$ The remaining $10 \%$ can be regarded as free. These are equilibrium estimates of the states of water at the micellar surface. There is of course dynamic exchange leading to such an equilibrium distribution.

As the micelle itself is undergoing slow rotational diffusion, some of the water molecules near the surface also experience this diffusion on a long time scale. These are the water molecules which have a very long residence time in the layer and are bound to the surface for a significant amount of the time, in addition to undergoing dynamic exchange. This model can be referred to as "ants on an elephant." So long as a given ant remains on or near the elephant surface for long enough time to experience the motion of the elephant, this will be reflected in the dynamics of the ant. A microscopic explanation of this, in terms of the mode coupling theory of correlated dynamics is given later.

Since the rotational diffusion of micelle is rather slow, our trajectory (3.4 ns long) catches only a part of this micellar rotational diffusion. Our preliminary calculation shows that the rotational time constant of CsPFO is about $5 \mathrm{~ns}$. We expect that bound water will get influenced but may not catch on to the full slowness because of the presence of water molecules' own dynamics. There is another important point to consider which is the amplitude of the 500 ps component. Since $90 \%$ of water molecules in the hydration layer are bound to the surface, the question naturally arises: why is its contribution $30 \%$ ? In order to understand this, we turn to the well-known mode coupling theory of relaxation in correlated systems. Let us first write the generalized Einstein relation for rotational diffusion

$$
D(z)=k_{B} T /[z+\zeta(z)],
$$

where $D(z)$ is the frequency dependent rotational diffusion coefficient and $\zeta(z)$ is the frequency dependent friction. The latter can be calculated from the torque-torque time correlation function

$$
\zeta(z)=\int_{0}^{\infty} d t \exp (-z t)\langle\mathbf{N}(0) \mathbf{N}(t)\rangle .
$$

The torque $\mathbf{N}(t)$ derives contributions not only from water molecules but also from the micellar atoms, in particular from the polar head groups, because of the large dipole moment of the water molecules. While the contributions from water molecules contribute at large frequencies, the ones from micelle contribute at very low frequencies because the random walk of micelles (leading to its Brownian rotational motion) occurs at a much longer time scale. In particular, we found that the micellar rotation is slowest along the axis with the largest dipole moment. This can give rise to a large contribution to the rotational friction because of the large contribution to the torque-torque time correlation function ex- pected from the interaction between the micellar dipole and the water dipole. Thus, $\zeta(z)$ is expected to have a rise at very small $z$ which will not be present in bulk water. This, we believe, could be the origin of the relatively large, $30 \%$ contribution of the 500 ps component.

The earlier analysis has further consequences. Several recent analyses of vibrational frequency shifts of $\mathrm{O}-\mathrm{H}$ and O-D stretch of water near protein surfaces have led to the suggestion that the water near such surfaces forms a percolating network characterized by very strong water-water hydrogen bonds, in addition to the $\mathrm{H}$ bonds to the charged/polar head groups of the protein. ${ }^{39}$ The existence of the ultraslow 500 ps component in the orientational relaxation of the water molecules seems to agree with such a picture, as discussed later. This conclusion is consistent with the observation made earlier that the hydrogen bond lifetime correlation function of the water molecules with the polar head group vary with the time resolution of study. Thus, the lifetime is of the order of a few picoseconds with a high resolution of $12 \mathrm{fs}$ but the lifetime extends to about 40 ps with the time resolution of 1 ps (see, for instance the $C_{\mathrm{HB}}(t)$ function in Ref. 16). This means that many of the hydrogen bonds that are broken are reunited with the same PHG in a very short time, without leaving the surroundings. This further implies the existence of a reinforcing network surrounding the water molecules. That is, the water molecules must remain close to the PHGs at the micellar surface sufficiently long enough to experience the torque due to the slow rotating micelle. Thus, while individual water molecules in the first layer are not exactly "sticking" to the micellar surface, they form a network which is long lived and rotate very slowly in the long time.

To summarize, the macro-microrelations show that the dielectric relaxation time can be several times larger than the orientational correlation time. Thus, it is fair to assume that it is the 19 ps time component in the single particle orientational correlation of water molecules at the micellar surface that gets elongated to the observed $41 \mathrm{ps}$ in the dielectric relaxation (see Table I). The 500 ps component in the reorientational TCF of water seems to arise from the coupling of water molecules in the layer to the micellar rotation.

The parameters obtained by fitting to the simulated $\Phi_{M}^{W}(t)$ using Eq. (6) can be used to calculate the frequency dependent dielectric function. We perform these calculations for the fitted $\Phi_{M}^{W}(t)$ function through Eq. (3) and display them in Figs. 8(a), 7(b), and 7(c). In Fig. 8(a), we show the frequency dependence of the real part, $\epsilon^{\prime}$ of the dielectric function. In Fig. 8(b), we show the same for the imaginary part. When compared with neat water, a clear shift of population to the slow frequency range is observed. The ColeCole plot shown in Fig. 8(c), shows considerable degree of nonexponentiality in the dielectric spectrum.

The main conclusion that emerges from all the earlier studies is the existence of a slow component in the 40-60 ps range in the dielectric relaxation. This slowness can be easily attributed to the slow rotation of surface water molecules. ${ }^{37}$ Our earlier studies have confirmed that this slow rotational relaxation is again due to the existence of quasibound state of water molecules due to hydrogen bonding to the nega- 

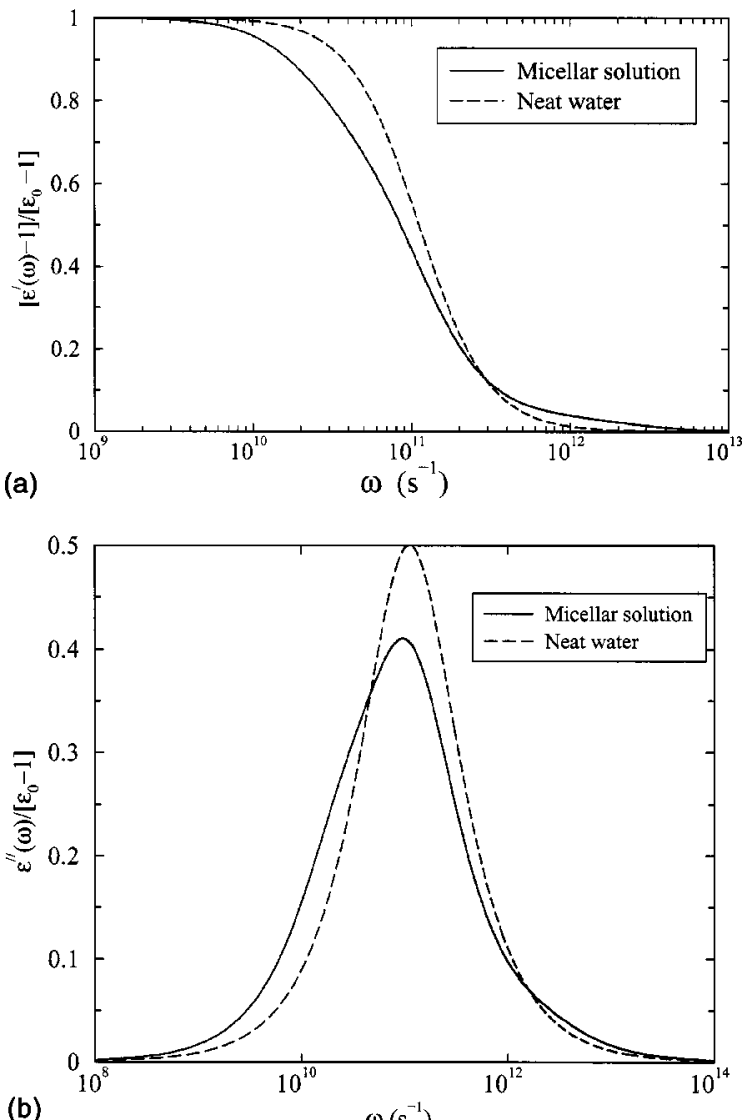

(b)

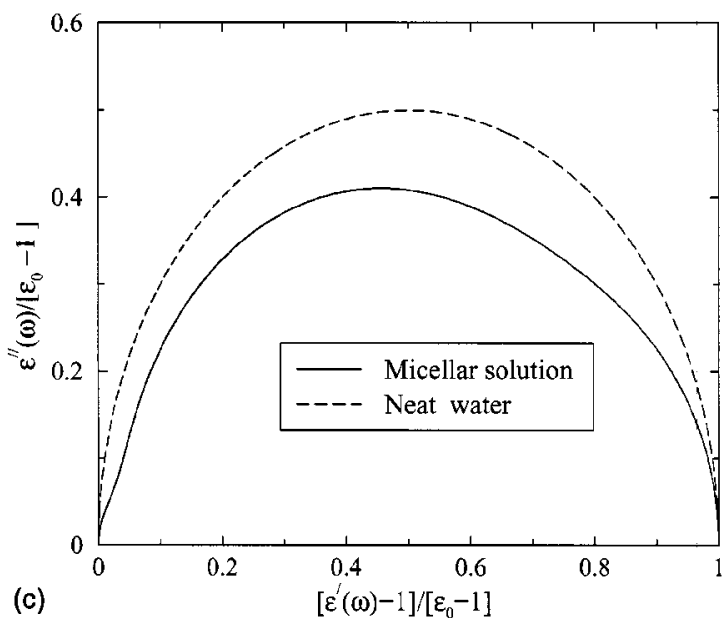

FIG. 8. (a) Real part of the frequency dependent dielectric function $\left\{\left[\epsilon^{\prime}(\omega)-1\right] /\left[\epsilon_{0}-1\right]\right\}$, for the water molecules, both in the micellar solution (solid line) and in neat water (dashed line). (b) Frequency dependence of the imaginary part of dielectric function $\left\{\left[\epsilon^{\prime \prime}(\omega)\right] /\left[\epsilon_{0}-1\right]\right\}$, for the water molecules, both in the micellar solution (solid line) and in neat water (dashed line). (c) The Cole-Cole plot of the frequency dependent dielectric function, $\epsilon(\omega)$, where the imaginary part, $\left[\epsilon^{\prime \prime}(\omega)\right] /\left[\epsilon_{0}-1\right]$ is plotted against the real part, $\left[\epsilon^{\prime}(\omega)-1\right] /\left[\epsilon_{0}-1\right]$, for the water molecules, both in micellar solution (solid line) and in neat water (dashed line).

tively charged oxygen atoms of the polar head group of the micelles.

\section{Relationship with water solvation dynamics at the interface}

The well-known continuum models and also the microscopic theories of solvation dynamics suggest a close rela- tion between solvation dynamics and dielectric relaxation. This is best expressed by the following relation between the longitudinal relaxation time $\tau_{L}$ and the dielectric relaxation time $\tau_{D}$ :

$$
\tau_{L}=\left(\frac{\epsilon_{\infty}}{\epsilon_{0}}\right) \tau_{D}
$$

However, the solvation dynamics of an ion at the surface of a micelle is difficult to describe theoretically because the ion faces a heterogeneous environment. Therefore, a straightforward application of the continuum model with a multiexponential description of dielectric relaxation is not possible. However, we can still derive certain semiquantitative conclusions from the existing theories. ${ }^{1}$ First, an initial ultrafast femtosecond decay will still arise from bulk water, and the slow decay should arise from nearest neighbor water molecules. The theory suggests that at short length scales, the relaxation time is essentially given by the dielectric relaxation time. ${ }^{40,41}$ Therefore, we certainly expect a slow component in the solvation dynamics with a time scale around 40 ps. As shown earlier, the decay of the equilibrium solvation time correlation function $C_{S}(t)$ of the natural probe $\mathrm{Cs}^{+}$ion indeed shows a pronounced component which is about 56 ps. ${ }^{15}$ Note that the earlier correspondence between solvation dynamics at micellar surface and dielectric relaxation of "biological water" was not feasible earlier in the absence of the dielectric relaxation study presented in this paper.

\section{CONCLUSIONS}

As described earlier, many experimental studies of dielectric relaxation in micelles and protein solutions have found a slow time component (in the 40-60 ps range) whose origin had not been fully understood. This component is sometimes referred to as $\delta$ dispersion. The amplitude of the decay is rather small and it often appears as a plateau between the two dominant relaxations due to bulk water and micelle/protein motions. ${ }^{1}$ It was proposed earlier that this 40 ps component denotes the existence of a dynamic equilibrium between the bound and free states of water molecules at a hydrophilic surface. The present simulations seem to further verify that picture.

It is indeed satisfying that the present study could detect the $40 \mathrm{ps}$ time component in the dielectric relaxation. As already emphasized, this appears to be a universal component observed in a large number of systems. Simulations, along with the well-known macro-microrelation, shows that this 40 ps component in DR originates from a 19 ps component in the orientational correlation function of the interfacial water molecules. We have recently observed the existence of this long time component in the dielectric relaxation of the solvent in a solution of a small protein. ${ }^{42}$

A somewhat unexpected but interesting finding of the present work is the presence of a 500 ps component in the orientational relaxation of the surface water molecules. We have used simulations and theoretical arguments to conclude that this ultraslow component arises at least partly due to the existence of network of water molecules at the micellar surface, and partly from the anisotropy in the orientation of the 
surfactant head groups. The latter could play a similar important role in the reorientation of water molecules on the surface of planar, lipid bilayers.

Future work should consider the contribution from the micelle and the charged groups in the frequency dependence of dielectric relaxation and also the effects of micellar rotation. Work in this direction is under progress.

\section{ACKNOWLEDGMENTS}

The research reported here was supported in parts by grants from the Council of Scientific and Industrial Research (CSIR), Department of Atomic Energy (DAE), and the Department of Science and Technology (DST), Government of India.

${ }^{1}$ N. Nandi, K. Bhattacharyya, and B. Bagchi, Chem. Rev. 100, 2013 (2000).

${ }^{2}$ E. H. Grant, V. E. R. McClean, N. R. V. Nightingale, R. J. Sheppard, and M. J. Chapman, Bioelectromagnetics (N.Y.) 7, 151 (1986); E. H. Grant, G. P. South, S. Takashima, and H. Ichimura, Biochem. J. 122, 691 (1971); C. G. Essex, E. H. Grant, R. J. Sheppard, G. P. South, M. S. Symonds, G. L. Mills, and J. Slack, J. Ann. N. Y. Acad. Sci. 303, 142 (1977).

${ }^{3}$ V. I. Gaiduk, Dielectric Relaxation and Dynamics of Polar Molecule (World Scientific, Singapore, 1999).

${ }^{4}$ S. Mashimo, S. Kuwabara, S. Yagihara, and K. Higasi, J. Phys. Chem. 91, 6337 (1987); M. Fukuzaki, N. Miura, N. Shinyashiki, D. Kurita, S. Shioya, M. Haida, and S. Mashimo, ibid. 99, 431 (1995).

${ }^{5}$ J. Berthel, R. Buchner, P.-N. Eberspächer, M. Müsterer, J. Stauber, and B. Wurm, J. Mol. Liq. 78, 83 (1998).

${ }^{6}$ C. Baar, R. Buchner, and W. Kunz, J. Phys. Chem. B 105, 2906 (2001).

${ }^{7}$ S. Boresch, P. Höchtl, and O. Steinhauser, J. Phys. Chem. B 104, 8743 (2000).

${ }^{8}$ S. Vajda, R. Jimenez, S. J. Rosenthal, V. Fidler, G. R. Fleming, and E. W. Castner, Jr., J. Chem. Soc., Faraday Trans. 91, 867 (1995).

${ }^{9}$ M. J. Lang, X. J. Jordanides, X. Song, and G. R. Fleming, J. Chem. Phys. 110, 5884 (1999); X. J. Jordanides, M. J. Lang, X. Song, and G. R. Fleming, J. Phys. Chem. B 103, 7995 (1999).

${ }^{10}$ K. Bhattacharyya, Acc. Chem. Res. 36, 95 (2003).

${ }^{11}$ S. K. Pal, J. Peon, and A. H. Zewail, Proc. Natl. Acad. Sci. U.S.A. 99, 1763 (2002).

${ }^{12}$ S. K. Pal, J. Peon, B. Bagchi, and A. H. Zewail, J. Phys. Chem. B 106, 12376 (2002).
${ }^{13}$ N. Nandi and B. Bagchi, J. Phys. Chem. B 101, 10954 (1997).

${ }^{14}$ S. Balasubramanian and B. Bagchi, J. Phys. Chem. B 106, 3668 (2002).

${ }^{15}$ S. Pal, S. Balasubramanian, and B. Bagchi, J. Chem. Phys. 117, 2852 (2002).

${ }^{16}$ S. Balasubramanian, S. Pal, and B. Bagchi, Phys. Rev. Lett. 89, 115505 (2002).

${ }^{17}$ C. D. Bruce, S. Senapati, M. L. Berkowitz, L. Perera, and M. D. E. Forbes, J. Phys. Chem. B 106, 10902 (2002).

${ }^{18}$ J. Faeder and B. M. Ladanyi, J. Phys. Chem. B 105, 11148 (2001).

${ }^{19}$ S. Senapati and M. L. Berkowitz, J. Chem. Phys. 118, 1937 (2003).

${ }^{20}$ M. Tarek and D. J. Tobias, Biophys. J. 79, 3244 (2000); A. R. Bizzarri and S. Cannistraro, J. Phys. Chem. B 106, 6617 (2002); M. Marchi, F. Sterpone, and M. Ceccarelli, J. Am. Chem. Soc. 124, 6787 (2002).

${ }^{21}$ J. G. Powles, J. Chem. Phys. 21, 633 (1953).

${ }^{22}$ A. Chandra and B. Bagchi, J. Phys. Chem. 94, 3152 (1990).

${ }^{23}$ N. Boden, K. W. Jolley, and M. H. Smith, J. Chem. Phys. 97, 7678 (1993).

${ }^{24}$ H. Iijima, T. Kato, H. Yoshida, and M. J. Imai, J. Phys. Chem. B 102, 990 (1998).

${ }^{25}$ H. J. C. Berendsen, J. R. Grigera, and T. P. Straatsma, J. Chem. Phys. 91, 6269 (1987).

${ }^{26}$ K. Watanabe and M. L. Klein, J. Phys. Chem. 95, 4158 (1991).

${ }^{27}$ M. Sprik, U. Röthlisberger, and M. L. Klein, Mol. Phys. 97, 355 (1999).

${ }^{28}$ M. E. Tuckerman, B. J. Berne, and G. J. Martyna, J. Chem. Phys. 97, 1990 (1992)

${ }^{29}$ M. E. Tuckerman, D. A. Yarne, S. O. Samuelson, A. L. Hughes, and G. Martyna, Comput. Phys. Commun. 128, 333 (2000).

${ }^{30}$ S. Balasubramanian and B. Bagchi, J. Phys. Chem. B 105, 12529 (2001).

${ }^{31}$ S. Balasubramanian, S. Pal, and B. Bagchi, Curr. Sci. 82, 845 (2002).

${ }^{32}$ R. Zwanzig, Annu. Rev. Phys. Chem. 61, 67 (1965).

${ }^{33}$ G. Williams, Chem. Rev. 72, 55 (1972).

${ }^{34}$ M. Neumann and O. Steinhauser, Chem. Phys. Lett. 106, 563 (1984).

${ }^{35}$ D. E. Smith and L. X. Dang, J. Chem. Phys. 100, 3757 (1994).

${ }^{36}$ S. Pal, S. Balasubramanian, and B. Bagchi, J. Phys. Chem. B 107, 5194 (2003).

${ }^{37}$ S. Pal, S. Balasubramanian, and B. Bagchi, Phys. Rev. E 67, 061502 (2003).

${ }^{38}$ B. Bagchi and A. Chandra, Adv. Chem. Phys. 80, 1 (1991).

${ }^{39}$ D. E. Khoshtariya, E. Hansen, R. Leecharoen, and G. C. Walker, J. Mol. Liq. 105/1, 13 (2003).

${ }^{40}$ P. G. Wolynes, J. Chem. Phys. 86, 5133 (1987).

${ }^{41}$ B. Bagchi, Annu. Rev. Phys. Chem. 4, 15 (1989).

${ }^{42}$ S. Balasubramanian, S. Pal, S. Bandyopadhyay, and B. Bagchi, Curr. Sci. 85, 101 (2003). 